\title{
Preface to the special collection in honor of Gregory S. Ezra
}

\author{
Srihari Keshavamurthy $\cdot$ Stephen Wiggins
}

Published online: 24 January 2015

(C) Springer-Verlag Berlin Heidelberg 2015

\section{A colleague's appreciation: Roger Loring, Cornell University}

As is customary in university departments, the doors of faculty offices in Cornell's Department of Chemistry and Chemical Biology indicate the name of the occupant. One of these doors, however, differs from the rest, lacking a name and decorated only with a small photograph of Rodin's Le Penseur. New arrivals to the department, from first-year undergraduates to faculty, soon learn that a thinker can indeed be found within.

Marking Greg Ezra's 60th birthday is also an occasion to recognize that half of that span has been spent at Cornell. Academic generations of undergraduate and graduate students have appreciated the clarity and rigor of his lectures, to say nothing of the preternaturally neat handwriting on the blackboard. A recent A. B. in chemistry who received an academic award wrote a letter of thanks to the benefactor who had endowed the prize. She chose to illustrate a high point of her undergraduate education with a drawing of Greg Ezra at the blackboard placing a "hat" on a letter $\mathrm{H}$. The intellectual incisiveness admired by students is highly valued by Greg's departmental colleagues in settings

Published as part of the special collection of articles celebrating the 60th birthday of Professor Greg Ezra.

S. Keshavamurthy $(\square)$

Department of Chemistry, Indian Institute of Technology,

Kanpur 208 016, India

e-mail: srihari@iitk.ac.in

\section{S. Wiggins $(\square)$}

Department of Mathematics, University of Bristol,

Bristol BS8 1TW, UK

e-mail: stephen.wiggins@me.com varying from informal consultations and formal research presentations to committee and faculty meetings. His Cornell colleagues are delighted to join with former and present collaborators, with contributors to this Festschrift, and with theoretical chemists generally in celebrating Greg Ezra's accomplishments and contributions to the field and community.

\section{A collaborator's appreciation: Stephen Wiggins, University of Bristol}

I have known Greg Ezra for some time, but we have only been seriously collaborating together for the past 7 years or so. I began graduate school at Cornell in 1983 and Greg had already been there for a year as an assistant professor of chemistry. He had recently taken on his first students (Larry Fried and Craig Martens, who have both contributed to this Festschrift). In those days, Cornell was one of the world's hot spots for the development of nonlinear dynamics from the theoretical, applied, and experimental perspectives. There was a sense of something new happening. Guckenheimer and Holmes had just been published and Phil Holmes was teaching his first course from that book, John Hubbard was teaching ordinary differential equations from $\mathrm{V}$. I Arnold's advanced ordinary differential equations book, Eric Siggia was teaching mechanics from V. I. Arnold's classical mechanics book, and Michael Fisher was lecturing on renormalization group theory. It was a time when everybody went to everybody's seminars, and I saw Greg at many of these seminars, discussing with his students. It seems to me that the Cornell environment had a significant influence on the development of Greg's research career in subsequent years. But it did not work out to be the right time for us to begin any type of collaboration since we both had more 
pressing concerns (he had to get tenure, and I had to pass qualifying exams). Even though we both went our separate ways, I continued to follow Greg's work over the years, and for reasons, I cannot remember, I sent him an e-mail in 2008 asking some question (that I also cannot remember). This was the start of a very rewarding collaboration that probably could not have happened earlier because I had to develop the type of mathematics that I was doing to a point where it might actually provide useful insight for a problem in chemistry, and I also needed to develop a common language so that I could benefit from his extensive knowledge. This took about 15 years, but it eventually got there.

Working with Greg is the ideal model of what someone would term an interdisciplinary collaboration (although I am not sure either of us have thought of it in that way). I have learned an incredible amount from working with him, and not just about chemistry. His encyclopedic knowledge of classical and quantum mechanics has greatly enriched our work. (It is a running joke now when we write papers that Greg must be falling down on the job if there are less than 100 cited references). When you work with Greg on a project, you will know what you can, and cannot, understand, about the project when it is completed. He is uncompromising and rigorous in his approach to research, and I have appreciated, admired, and benefited from that greatly. But there are aspects to Greg's scholarship that are probably not so well known. Greg is a very serious scholar of the best of twentieth century music (and he can also convince you that vinyl provides a superior listening experience). For example, he can explain in detail the evolution of the drumming style of Ginger Baker, and he has a deep knowledge of the influence of American blues music on the great British rock music of the 1960's.

I am sorry that I did not get to know Greg during my days as a graduate student. But everything has its time, and I feel fortunate that I now have this collaboration, and I wish Greg the best for the next 60 years.

\section{A former postdoc's appreciation: Srihari Keshavamurthy, IIT Kanpur}

I had the honor of joining Greg Ezra as a fresh postdoc in the winter of 1995. In fact, having met and discussed briefly with Greg on semiclassics in the 1993 Seattle APS meeting, I was convinced that Greg was the person I wanted to work for my postdoc. I have not regretted that decision! Coming from Berkeley, the recent snowstorm in Ithaca was quite an experience for me. I had to meet Greg at his office in the morning and did so by trudging through snow from the motel to Baker laboratories. Two things that I remember from this first meeting-first, I was the only occupant of that part of Baker laboratory and second, Greg had already prepared a list of papers concerned with dynamical tunneling. Greg told me that he would like to understand dynamical tunneling and its influence on molecular spectra and asked me to analyze the highly excited vibrational states of an effective Hamiltonian for water. I remember being immersed in calculating surfaces of sections, computing resonance zones and widths and looking at hundreds of vibrational eigenstates. Interestingly, and thankfully, Greg let me pursue my line of thoughts on the dynamical assignment problem. At the same time, through numerous discussions and gracefully tolerating many of my grandiose ideas, Greg passed on to me his immense knowledge and deep insights into the phenomenon of intramolecular vibrational energy redistribution. I was amazed by Greg's ability to point out a relevant paper irrespective of when it was published-sometimes a hard copy would emerge from one of the stacks on his desk. Equally striking was the fact that he had not only read these papers through, but also had a firm grasp of the limitations of the approach used in these works. This was an important and a very valuable lesson for me and, for that matter, any honest academician. For those who have had the pleasure of reading Greg's papers, it is a bit unnerving to realize that he has most probably read every one of the hundreds of cited references.

An event that occurred within a few months of my coming to Ithaca has left a lasting impression on me in terms of my current research interests. Greg asked me to attend a NATO ASI workshop on Hamiltonian systems with three or more degrees of freedom in June 1995 held in S'Agaro, Spain. This was a one of a kind meeting and a heady one for a young person with people like Arnold, Moser, and Sinai being there. Attending this workshop exposed me to the great beauty of nonlinear dynamics and the challenges of applying them to molecular Hamiltonians-I am yet to recover from this meeting! But then, the challenges remain and it is a great pleasure to see Greg's recent works that take a step closer toward meeting these challenges. In any case, I am forever indebted to Greg for providing me with this unique opportunity.

My own research has benefited immensely from the beautiful insights into classical-quantum correspondence that have come from Greg's work over the years. Perhaps, to a small extent, I have imbibed even some of his writing skills - to quote Samuel Johnson 'language is the dress of thought.' A colleague read a preprint of mine and remarked that "It is a very scholarly piece of work. There is a Greg Ezra style infused in your work. This is good." I take that as a high compliment.

\section{A former graduate student's appreciation: Craig Martens, University of California, Irvine}

During the summer of 1982, I traveled from Nebraska to Ithaca, NY to begin my graduate studies in chemistry at 
Cornell University. I was drawn to that beautiful campus by its long history of being a home to luminaries of sciencefrom Debye and Feynman to the newly minted Nobel Laureate Roald Hoffmann and the television star Carl Saganbut it was another newcomer that would end up changing the course of my life. This was Greg Ezra, a new assistant professor with a small (and already cluttered) office and a cavernous laboratory equipped with an antique glove box and a single computer terminal. I remember that first talk in Greg's office about his research. Compact and quiet, with an unruly mop of black hair, Greg stood at the blackboard sketching invariant tori and writing equations with his neat handwriting, his eyes bright and intense. I was hooked.

And so began the graduate education of a theoretical chemist. At the time, I did not fully appreciate how special that education was to be. Greg the thesis advisor was not one to give orders or lecture pedantically to his students, and would not dream of being so presumptuous as to comment on ones work habits. Rather, he led by example. A consummate scholar, Greg seemed to have already read (and photocopied) everything. It was rare to visit Greg's office without him recalling a relevant article and extracting it, miraculously, from the center of one of the tall piles of papers that fought for space on every level surface. Greg was embarking on a new research direction, combining semiclassical mechanics with nonlinear dynamics, and was more concerned about learning this new field than about showing off what he knew. He encouraged a continual commitment to intellectual development by attending seminars and reading literature, and made it possible for his students to attend conferences and workshops. He would even go with us to sit in on classes in other departments. And although he did not have a big ego, he set high standards. Greg demanded rigor in science and scholarship, but also for usage of the English language. Writing papers with him was an intense process, sometimes requiring a trip to his office to consult his immense copy of the Oxford Dictionary to determine the fate of a single word.

There were a few things that Greg did not teach his students. Things like overselling your work, ignoring or minimizing previous contributions by others, cranking out numerous mediocre publications, navigating the meta-scientific waters of politics and fashion, and the tireless pursuit of credit and citation. To Greg, the scientists life is one driven by a passion to appreciate the beautiful whole that emerges by seeing the connections between ideas-yours and those of a community of scholars. He taught by example the ideals of hard work, intellectual and personal honesty, optimism, generous sharing of credit with others, and the final authority of the truth and beauty of natural law. I have become older and wiser since leaving Ithaca. But the essential principles and practices that set the high bar I believe a scientist should strive for are things I picked up (and hopefully have not misplaced) long ago from Greg Ezra.

\section{Greg Ezra: Scientific autobiography}

\section{Ed é subito sera ${ }^{1}$}

Ognuno sta solo cuor della terra

traffito da un raggio di sole:

ed é subito sera

Salvatore Quasimodo (1901-1968)

\subsection{Beginnings}

I was born and raised in northwest London. A bookish and intellectually precocious child, I early on developed the habit of amassing and organizing large amounts of data and information in order to write my own 'encyclopedias'; some of my coworkers will perhaps recognize this trait!

Throughout the course of my education in the UK, I was extremely fortunate to have benefitted from the state run system in place at the time that recognized ability and aptitude in children of modest backgrounds, and facilitated their passage to academically excellent schools. So, I passed from Braintcroft Primary in Neasden to Merchant Taylors' (alma mater of, among others, Edmund Spenser, Clive of India and Boris Karloff) to St. John's college, Oxford, as a Thomas White Scholar. At Merchant Taylors', I received much support and encouragement from several inspiring teachers; in particular, John Dishman and Andy Edge (chemistry), and Alan Whitney (art).

\subsection{Apprenticeship}

I studied chemistry at Oxford, with equal time devoted to Organic, Inorganic and Physical branches. It became clear early on that I was not cut out to be an experimentalist (despite having a strong interest in synthetic organic chemistry in high school), and so, it was necessary to pick up the mathematics and physics necessary for deeper study of theory on my own initiative.

A first encounter with transition state theory as an undergraduate set the stage for decades of interest in this fascinating topic: After I complained to my tutor that the theory made absolutely no sense (What, precisely, were the assumptions involved? Where, exactly, had the dynamics gone?), it was suggested that I write a research paper to clarify my misgivings on the subject! At this point, the

\footnotetext{
${ }^{1}$ And suddenly it's evening Everyone stands alone at the heart of the world, pierced by a ray of sunlight: and suddenly it's evening Salvatore Quasimodo (1901-1968)
} 
seminal papers of Pechukas and Pollak on periodic orbit dividing surfaces, etc, had not appeared, and I had yet to read Wigner's remarkably lucid treatment of the topic.

Peter Atkins had a profound influence on my intellectual direction through both his textbooks and lectures on quantum mechanics. I therefore joined Peter's research group for my part II (a thesis on multiphoton processes, leading to a lifelong interest in angular momentum and symmetry) and my Ph.D. thesis.

As a Senior Scholar at Christ Church, Oxford, I had the freedom to chart my own course as a graduate student. My research direction was set after I heard an inspiring talk by Brian Sutcliffe on the properties of the Eckart frame (used to separate molecular vibration from rotation), in particular the connection with molecular symmetry properties. This talk was based both on Brian's own work and on the thenrecent review by Louck and Galbraith. Already familiar with the Eckart-Sayvetz frame, which generalizes the Eckart frame to non-rigid molecules, I resolved to carry out the same kind of analysis for non-rigid systems. (I was unaware that very similar work was being undertaken by Natanson and Adamov in the Soviet Union at about the same time). Extensive use was made of the theory of induced representations, which I learned from the book of Simon Altmann and the papers and lectures of Harald Fritzer. This work formed the basis for my D. Phil. thesis, and for my continuing interest in the subtleties associated with use of the Eckart condition and with vibration-rotation interaction generally. The puzzling (to me, at the time) observations of Meyer and Günthard on the non-integrability of the conditions for removal of rotation-vibration coupling and many other outstanding problems besides were eventually beautifully elucidated by the seminal work of Guichardet, Iwai, Tachibana and Littlejohn on holonomy in the RV problem.

I had the good fortune to meet Steve Berry during one of his visits to Oxford and went to Chicago to work with Steve as a NATO Postdoc. At Chicago, I worked on a number of projects related to the properties of "floppy molecules" which, following the seminal work of Kellman and Herrick, included the doubly excited Helium atom! While at Chicago I learned how to program a trajectory integration algorithm, and so began to explore the classical mechanics of the Helium atom. Influenced by the work of Percival and others on semiclassical quantization of vibrational levels, my (very näive) plan was to find invariant tori in the Helium atom phase space, and hence obtain quantized levels for 2-electron systems. I very quickly discovered that any randomly selected non-symmetric Helium atom trajectory will apparently autoionize with probability one (!), and that singular Coulomb potentials require application of special but well-established regularization techniques. So, began a long-term interest in the classical and semiclassical mechanics of few-body Coulomb systems, which was to culminate in a few years in the semiclassical quantization of the He atom.

\subsection{Vocation}

After Chicago, the plan was to return to an academic post in the UK, but the situation in the job market dictated otherwise. I interviewed for a faculty position at a number of US universities, and was offered a job at Cornell, located somewhere in upstate NY in a place called Ithaca. There I have been ever since.

My research at Cornell has covered many aspects of the few-body problem in chemistry and physics. In broad terms, I have been interested in the role of symmetry in physical problems, in classical, semiclassical and quantum approaches to atomic and molecular systems, and in geometrical aspects of Hamiltonian and non-Hamiltonian dynamics.

I have been fortunate to have had a number of talented and creative students and postdocs over the years, several of whom have gone on to distinguished careers in research. I count myself particularly lucky that my first graduate students were Craig Martens and Larry Fried, both of whom worked on problems in semiclassical mechanics. Craig's work on the Fourier quantization of quasiperiodic trajectories in multidimensional systems led us to inquire what power spectra looked like for finite time segments of nonregular trajectories; the resulting method of 'Local Frequency analysis' (a collaborative effort with Mike Davis) has been developed and applied to a number of physical problems since by many others since. Larry's work focussed on the use of Lie-transform perturbation theory to derive simplified Hamiltonians for molecular vibrational problems, in the spirit of earlier work of Swimm-DelosJaffé-Shirts-Reinhardt; this work led to an interest in the symplectic geometry of phase space which has informed much of my work since.

A turning point in the Helium atom problem came when I attended a Cornell seminar given by Robert Devaney, who talked about the application of the McGehee 'blow-up' technique to the analysis of the dynamics of the anisotropic Kepler problem. (Steve Wiggins, then a graduate student at Cornell, was also at this very memorable talk.) I was aware that Gutzwiller's periodic orbit quantization technique had been applied to the anisotropic Kepler problem, which was conjectured to be completely chaotic, but was unfamiliar with the various blow-up and regularization techniques used in celestial mechanics. Regularization of pairwise collisions would facilitate numerical integration of $\mathrm{He}$ atom trajectories, while analysis of the triple collision manifold would perhaps provide the basis for application of some kind of symbolic dynamics. Numerical work on a (suitably regularized) collinear model for the classical $\mathrm{He}$ atom 
showed that these approaches worked well and suggested that the system might be completely chaotic, with unstable periodic orbits coded by a complete binary code. My student Jong-Hyun Kim carried out extensive computations of doubly excited resonances in the collinear model, so that by applying the Gutwiller trace formula 'in reverse,' we were able to use the scaled quantum levels to extract information on the nature of the classical periodic orbits contributing to the quantized level structure. Our results definitively showed that it is the 'antisymmetric stretch' po, not the 'symmetric stretch' po, as had previously been thought, that is the most important po associated with the manifold of $n=n^{\prime}$ doubly excited states. The symmetric stretch orbit is in a sense infinitely unstable, and hence does not contribute to the semiclassical spectrum, although it serves as the basis for organizing the classical phase space structure.

These results were presented at the 1990 Trieste conference on Quantum Chaos and published in the proceedings. (A short paper describing these findings was rejected by Physical Review Letters; a full account is given in Kim's unpublished doctoral thesis.) At the Trieste meeting, I was fortunate to meet the late Dieter Wintgen and his students Grigor Tanner and Klaus Richter, who were working on the same problem. They had been using similar techniques to analyze the classical dynamics of $\mathrm{He}$, and I ended up as a co-author on a short paper describing the application of the cycle-expansion technique to the more difficult problem of forward quantization of the He. While this work overturned textbook dogma about the inapplicability of semiclassical quantization to the 2-electron problem, it of course relied on more than a half century of development of semiclassics and classical dynamics! It is also true to say that many aspects of the phase space structure of the 3D He atom remain to be understood.

The problem of comprehending dynamics in multidimensional systems (that is, those with $N \geq 3$ degrees of freedom) has been a central theme of my research. In 1991, Richard Gillilan and I published a study of dividing surfaces and 'turnstiles' in a 4D model of a van der Waals complex dissociation reaction (as a symplectic mapping). One surprise was the possibility of the non-existence of turnstiles (phase space structures mediating passage between bound and unbound regions) in higher dimensions. This work was inspired by a seminal paper of Steve Wiggins on Normally Hyperbolic Invariant Manifolds (NHIMs), which has provided the foundation for much subsequent research in the field.

Another noteworthy excursion into higher dimensional phase space was the study of the Baggot Hamiltonian for the water molecule, carried out in collaboration with Srihari Keshavamurthy.

In the past few years, I have enjoyed a very stimulating and productive collaboration with Steve Wiggins, who is in the Mathematics department at Bristol University, and other members of the 'Bristol group'. We have worked on a number of topics, such as thermostats, higher-index saddles, transition state theory and the gap time formalism, the roaming mechanism, and non-statisticality on organic reaction mechanisms. A major theme of this work is the important role played by NHIMs and related phase space structures in reaction dynamics, long emphasized by Wiggins, and the importance of adopting a phase space perspective for understanding mechanisms and non-statisticality in general. I am particularly pleased to be currently working on the theory of the dynamics of organic reactions with my former Cornell colleague Barry Carpenter, now at Cardiff University, and on roaming dynamics with Stavros Farantos (Iraklion, Greece), whose work on periodic orbit theory I have followed for a long time.

Space does not allow full discussion of the various other projects and topics of interest we have investigated over the years: non-adiabatic dynamics; dynamics of ion-molecule reactions; rotation-vibration interactions; semiclassics for singular potentials; periodic orbit analysis of vibrational spectra; semiclassical theory of nonlinear response functions; geometrical approach to non-Hamiltonian (thermostat) dynamics; quantum monodromy in molecular rotors.

\subsection{Avocation}

When not teaching or researching, I can often be found playing and performing music as a drummer-percussionist, pursuing anew a youthful interest in the percussive arts. I enjoy playing in a variety of genres such as blues, folkrock, Middle Eastern and Klezmer music. Making live music in real time with real people is to me a perfect antidote to the alienating and (dis)connected nature of our digital world.

\subsection{Sine qua non}

The Cornell Chemistry (now Chemistry and Chemical Biology) department is an extraordinary place, and I am singularly fortunate to have been able to spend my career as an independent scientist there. Inhabited by titans such as (the late) Andy Albrecht, Michael Fisher, Jack Freed, Roald Hoffmann, Harold Scheraga, and Ben Widom (to name but a few), and dynamic younger theorists Nandini Ananth, Garnet Chan (now at Princeton), and Roger Loring, it is a uniquely collegial and friendly department. Most important, the department as a whole has a genuine and unwavering commitment to the highest standards of intellectual rigor and scholarship, regardless of research fads or fashions, and to quality teaching. It is a wonderfully supportive environment in which to pursue research on fundamental problems in chemical physics. 
I offer my sincere gratitude to all my coworkers past and present for their inspiration and efforts, and especially those friends, colleagues, and collaborators (by no means mutually exclusive categories!) who have contributed their excellent papers to this special issue of TCA. Particular thanks are due to Srihari Keshavamurthy and Steve Wiggins for devoting their time and energy to organizing this Fest. It is indeed humbling to be honored in this way.

Finally, I can hardly begin to thank my wife Sally for many years of support and companionship and for her loving forebearance toward an often-distracted thinker of 'deep thoughts', and our daughters Rachel and Ruth for having enriched our lives immeasurably.

\section{Publications of Gregory S. Ezra ${ }^{2}$}

1. G.S. Ezra, "On the symmetry properties of nonrigid molecules: a unified approach", Mol. Phys. 38, 863875 (1979).

2. G.S. Ezra, "On the symmetry properties of nonrigid molecules:semifactorizability of the isometric group", Mol. Phys. 43, 773-783 (1981).

3. G.S. Ezra, "Symmetry properties of molecules", Lecture Notes in Chemistry 28 (Springer, 1982).

4. G.S. Ezra, "Symmetry properties of nonrigid molecules", in: Proc. Bielefeld Conf. Molecular Structure, Rigidity and Potential Energy Surfaces, 1980, Ed. J. Hinze (Plenum, 1983).

5. G.S. Ezra, "The semidirect product structure of nonrigid molecule symmetry groups", in: Proc. Intl. Conf. Symmetry and Properties of Nonrigid Molecules, Ed. J. Maruani (Elsevier, 1983).

6. H.J. Yuh, G. Ezra, P. Rehmus and R.S. Berry, "Electron correlation and Kellman-Herrick quantization in doubly-excited Helium”, Phys. Rev. Lett. 47, 497 (1981).

7. G.S. Ezra and R.S. Berry, "Correlation of two particles on a sphere", Phys. Rev. A 25, 1513-1527 (1982).

8. G.S. Ezra and R.S. Berry, "Correlation diagrams for rigid and nonrigid five-body and $X Y_{5}$ six-body systems", J. Chem. Phys. 76, 3679-3691 (1982).

9. R.S. Berry, G.S. Ezra and G.A. Natanson, "Collective and independent particle motion in simple atoms and molecules: a unification", in: Proc. 4th Intl. Cong. Quantum Chem. 1982 (Reidel, 1983).

10. G.S. Ezra and R.S. Berry, "Collective and independent-particle motion in doubly-excited two-electron atoms", Phys. Rev. A 28, 1974-1988 (1983).

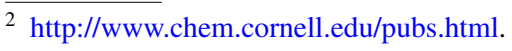

11. G.S. Ezra and R.S. Berry, "The quantum states of two particles on concentric spheres", Phys. Rev. A 28, 1989-2000 (1983).

12. G.S. Ezra and R.S. Berry, "Comment on: 'Classification of doubly-excited states of two-electron atoms", Phys. Rev. Lett. 52, 1252-1252 (1984).

13. G.A. Natanson, G.S. Ezra, G. Delgado-Barrio and R.S. Berry, "Calculation of rovibrational spectra of water by means of particles-on-concentric-spheres models. I. Ground stretching vibrational state", J. Chem. Phys. 81, 3400-3406 (1984).

14. G.A. Natanson, G.S. Ezra, G. Delgado-Barrio and R.S. Berry, "Calculation of rovibrational spectra of water by means of particles-on-concentric-spheres models. II. Excited stretching vibrational states", J. Chem. Phys. 84, 2035-2044 (1986).

15. G.S. Ezra, "The adiabatic approximation for coupled oscillators", Chem. Phys. Lett. 101, 259-264 (1983).

16. R.S. Tapper, R.L. Whetten, G.S. Ezra and E.R. Grant, "The role of near-resonant intermediate states in twophoton excitation $\mathrm{NO}_{2}$ : origin bands in bent-to-linear transitions", J. Phys. Chem. 88, 1273-1275 (1984).

17. C.C. Martens and G.S. Ezra, "A simple method for determining the number of isolating integrals in multidimensional systems: computation of the pointwise dimension”, Chem. Phys. Lett. 108, 573-579 (1984).

18. R.L. Whetten, G.S. Ezra and E.R. Grant, "Molecular dynamics beyond the adiabatic approximation: new experiments and theory", Ann. Rev. Phys. Chem. 36, 277-320 (1985).

19. J. Zwanziger, R.L. Whetten, G.S. Ezra and E.R. Grant, "Semiclassical eigenvalues for a nonadiabatic system", Chem. Phys. Lett. 120, 106-112 (1985).

20. C.C. Martens and G.S. Ezra, "EBK quantization of nonseparable systems: a Fourier transform method", J. Chem. Phys. 83, 2990-3001 (1985).

21. L. Bigio, G.S. Ezra and E.R. Grant, "Intramolecular dynamics and multiresonant absorption spectroscopy. II. Power broadening and superposition states in double resonant two-photon excitation", J. Chem. Phys. 83, 5369-5379 (1985).

22. E.M. Goldfield, P.L. Houston and G.S. Ezra, "Nonadiabatic interactions in the photodissociation of ICN", J. Chem. Phys. 84, 3120-3129 (1986).

23. J. Santamaria, G. Alvarez Galindo, R. Escribano and G.S. Ezra, "Rotation-vibration separability in the classical motion of triatomics", J. Mol. Struct. 142, 529-532 (1986).

24. G.S. Ezra, "Interaction between bending vibrations and molecular rotations: a model study", Chem. Phys. Lett. 127, 492-500 (1986).

25. J. Zwanziger, E.R. Grant and G.S. Ezra, "Semiclassical quantization of a classical analogue for the Jahn- 
Teller Exe system”, J. Chem. Phys. 85, 2089-2098 (1986).

26. C.C. Martens and G.S. Ezra, "Uniform quantization of multidimensional systems", in: Proc. 19th Jerusalem Symp. Quantum Chem., Tunnelling, Ed. J. Jortner and B. Pullman (Reidel, 1986).

27. C.C. Martens and G.S. Ezra, "Semiclassical mechanics of strongly resonant systems: a Fourier transform approach", J. Chem. Phys. 86, 279-307 (1987).

28. L. Fried and G.S. Ezra, "PERTURB: a specialpurpose algebraic manipulation program for classical perturbation theory", J. Comp. Phys. 8, 397-411 (1987).

29. L. Fried and G.S. Ezra, "Semiclassical quantization using perturbation theory: algebraic quantization of multidimensional systems", J. Chem. Phys. 86, 62706282 (1987).

30. C.C. Martens and G.S. Ezra, "Classical, quantum mechanical and semiclassical representations of resonant dynamics: a unified treatment", J. Chem. Phys. 87, 284-301 (1987).

31. G.S. Ezra, C.C. Martens and L.E. Fried, "Semiclassical quantization of polyatomic molecules: some recent developments", J. Phys. Chem. (Invited Feature Article) 91, 3721-3730 (1987).

32. A. Garcia-Ayllon, C.C. Martens, J. Santamaria and G.S. Ezra, "Semiclassical vibrational transition frequencies for a Hamiltonian with stretch-bend potential energy coupling: application of Fourier methods", J. Phys. Chem. 87, 6609-6617 (1987).

33. B.G. Sumpter and G.S. Ezra, "Semiclassical rotationvibration energies for a triatomic molecule: $\mathrm{H}_{2} \mathrm{O}$ ", Chem. Phys. Lett. 142, 142-146 (1987).

34. C.C. Martens, M.J. Davis and G.S. Ezra, "Local frequency analysis of chaotic motion in multidimensional systems: energy transport and bottlenecks in planar OCS", Chem. Phys. Lett. 142, 519-528 (1987).

35. L.E. Fried and G.S. Ezra, "Generalized algebraic quantization: corrections to arbitrary order in $\hbar$ ", $\mathrm{J}$. Phys. Chem. 92, 3144-3154 (1988).

36. L.E. Fried and G.S. Ezra, "PERTURB: A program for calculation of vibrational energies using generalized algebraic quantization", Comp. Phys. Comm. 51, 103-114 (1988) [Invited contribution to Special Issue on Molecular Vibrations].

37. A. Garcia-Ayllon, J. Santamaria and G.S. Ezra, "Sensitivity of IVR to stretch-bend potential energy coupling and stability of periodic orbits", J. Chem. Phys. 89, 801-811 (1988).

38. B.G. Sumpter, C.C. Martens and G.S. Ezra, "Interaction of molecular rotation with large-amplitude internal motions: a rigid twister model for hydrogen peroxide”, J. Phys. Chem. 92, 7193-7204 (1988).
39. L.E. Fried and G.S. Ezra, "Avoided crossings and resummation of nearly resonant molecular vibrations: reconstruction of an effective secular equation", J. Chem. Phys. 90, 6378-6390 (1989).

40. C. Getino, B.G. Sumpter, J. Santamaria and G.S. Ezra, "Unimolecular decay lifetimes and intramolecular energy redistribution in $\mathrm{HOOH}$ : sensitivity to potential energy surface", J. Phys. Chem. 93, 38773880 (1989).

41. R.M. Benito, F. Borondo, J.H. Kim, B.G. Sumpter and G.S. Ezra, "Comparison of classical and quantum phase space structure of nonrigid molecules: LiCN", Chem. Phys. Lett. 161, 60-66 (1989).

42. C. Getino, B.G. Sumpter, J. Santamaria and G.S. Ezra, "Ab initio study of stretch-bend coupling in HOOH”, J. Phys. Chem. 94, 3995-4000 (1990).

43. R.E. Gillilan and G.S. Ezra, "Turnstiles, transport and unimolecular decay in multidimensional Hamiltonian maps: application to van der Waals predissociation", J. Chem. Phys. 94, 2648-2668 (1991).

44. J.H. Kim and G.S. Ezra, "Periodic orbits and the classical-quantum correspondence for doubly-excited states of two-electron atoms", Proc. Trieste Workshop Quantum Chaos, pp 436-448, Ed. H. Caldiera, M. Gutzwiller and R. Ramaswamy (World Scientific, 1991).

45. G.S. Ezra, K. Richter, G. Tanner and D. Wintgen, "Semiclassical cycle expansion for the Helium atom", J. Phys. B 24, L413-L420 (1991).

46. G.S. Ezra, "Classical trajectory studies of intramolecular dynamics: local mode dynamics, rotation-vibration interaction and the structure of multidimensional phase space", in Intramolecular Dynamics and Nonlinear Dynamics, pp 1-40, edited by W.L. Hase (JAI Press, 1992).

47. G. Ramachandran and G.S. Ezra, "Vibrational deactivation in planar ion-neutral collisions: a classical trajectory study of the fixed-angle approximation", Chem. Phys. Lett. 199, 397-402 (1992).

48. G. Ramachandran and G.S. Ezra, "Vibrational deactivation in $\mathrm{Kr} / \mathrm{O}_{2}^{+}$collisions: role of complex formation and potential anisotropy", J. Chem. Phys. 97, 63226334 (1992).

49. H. Li, G.S. Ezra and L.A. Philips, "Vibrationally induced axis switching: a novel mechanism for vibration-rotation coupling", J. Chem. Phys. 97, 59565963 (1992).

50. K.M. Atkins and G.S. Ezra, "Semiclassical density of states at symmetric pitchfork bifurcations in coupled quartic oscillators" Phys. Rev. A 50, 93-97 (1994).

51. R. Manning and G.S. Ezra, "A regularized semiclassical radial propagator for the Coulomb potential", Phys. Rev. A 50, 954-966 (1994). 
52. R.J. Hinde and G.S. Ezra, "Direct and complex forming collisions in a model for the barrierless proton transfer reaction: $\mathrm{CH}_{3} \mathrm{OH}+{ }^{-} \mathrm{OCH}_{3} \rightarrow \mathrm{CH}_{3} \mathrm{O}^{-}+\mathrm{HOCH}_{3}$ ", Chem. Phys. Lett. 228, 333-340 (1994).

53. G. Ramachandran and G.S. Ezra, "Orbiting complex formation in $\mathrm{Na}^{+} / \mathrm{N}_{2}$ collisions: a phase space view"[Invited contribution to S. Rice issue], J. Phys. Chem. 99, 2435-2443 (1995).

54. K.M. Atkins and G.S. Ezra, "Classical-quantum correspondence and the transition from regular to chaotic dynamics in coupled quartic oscillators", Phys. Rev. E 51, 1822-1837 (1995).

55. D.C. Rouben and G.S. Ezra, "Periodic orbit analysis of molecular vibrational spectra: 1:1 resonant coupled modes", J. Chem. Phys. 103, 1375-1383 (1995).

56. G.S. Ezra, "Periodic orbit analysis of molecular vibrational spectra: Spectral patterns and dynamical bifurcations in Fermi resonant systems", J. Chem. Phys. 104, 26-35 (1996).

57. R. Manning and G.S. Ezra, "A uniform regularized semiclassical propagator for the $1 / x^{2}$ potential", Phys. Rev. A 53, 661-668 (1996).

58. S. Keshavamurthy and G.S. Ezra, "Analysis of quantum eigenstates in a 3-mode system", in Proc. NATO ASI on Hamiltonian Systems with Three or More Degrees of Freedom, p 435-439, ed C. Simo, (Kluwer, 1999).

59. S. Keshavamurthy and G.S. Ezra, "Assigning vibrational spectra of highly-excited molecules: Classical and quantum vibrational dynamics of the $\mathrm{H}_{2} \mathrm{O}$ molecule", Chem. Phys. Lett. 259, 81-90 (1996).

60. S. Keshavamurthy and G.S. Ezra, "Eigenstate assignments and the quantum-classical correspondence for highly-excited states of the Baggot $\mathrm{H}_{2} \mathrm{O}$ Hamiltonian", J. Chem. Phys. 107, 156-179 (1997).

61. G.S. Ezra, "Classical-quantum correspondence and the analysis of highly-excited states: periodic orbits, rational tori and beyond", in Advances in Classical Trajectory Methods, Vol. 3, pp 35-72, edited by W.L. Hase (JAI Press, 1998).

62. C.C. Martens, M.J. Davis and G.S. Ezra, "Comment on 'Local Frequency analysis and the structure of classical phase space of the $\mathrm{LiNC} / \mathrm{LiCN}$ molecular system' [J. Chem. Phys. 108, 63 (1998)], J. Chem. Phys. 109, 6507 (1998).

63. M. Tsuchiya and G.S. Ezra, "Global analysis of periodic orbit bifurcations in coupled Morse systems: Time-reversal symmetry, permutational representations and codimension-2 collisions", CHAOS 9, 819840 (1999).

64. G. S. Ezra, "Geometric approach to response theory in non-Hamiltonian systems", J. Math. Chem. 32, 339-359 (2002).
65. G. S. Ezra, "On the statistical mechanics of non-Hamiltonian systems: the generalized Liouville equation, entropy, and time-dependent metrics", J. Math. Chem. 35, 29-53 (2004).

66. W. G. Noid, G. S. Ezra, and R. F. Loring, "Optical response functions with semiclassical dynamics", J. Chem. Phys. 119, 1003-1020 (2003).

67. W. G. Noid, G. S. Ezra, and R. F. Loring, "Vibrational echoes: Dephasing, rephasing, and the stability of classical trajectories", J. Phys. Chem. 108, 65366543 (2004)

68. W. G. Noid, G. S. Ezra, and R. F. Loring, "Semiclassical calculation of the vibrational echo", J. Chem. Phys. 120, 1491-1499 (2004).

69. C. A. Arango, W. W. Kennerly, and G. S. Ezra, "Quantum monodromy for diatomic molecules in combined electrostatic and pulsed nonresonant laser fields",Chem. Phys. Lett. 392, 486-492 (2004).

70. C. A. Arango, W. W. Kennerly, and G. S. Ezra, "Quantum and classical mechanics of diatomic molecules in tilted fields",J. Chem. Phys. 122, Art. No. 184303 (2005).

71. C. A. Arango, W. W. Kennerly, and G. S. Ezra,"Semiclassical IVR approach to rotational excitation of nonpolar diatomic molecules by nonresonant laser pulses",Chem. Phys. Lett. 420, 296-303 (2006).

72. S. A. Deshpande and G. S. Ezra, "On the derivation of the Herman-Kluk propagator”,J. Phys. A 39, 50675078 (2006).

73. G. S. Ezra, "Reversible measure-preserving integrators for non-Hamiltonian systems",J. Chem. Phys. 125, Art. No. 034104 (2006).

74. S. A. Deshpande and G. S. Ezra, "Quantum state reconstruction for rigid rotors",Chem. Phys. Lett. 440, 341-347 (2007).

75. C. A. Arango and G. S. Ezra, "Classical mechanics of dipolar asymmetric top molecules in collinear static electric and nonresonant linearly polarized laser fields:energy-momentum diagrams, bifurcations and accessible configuration space",Int. J. Bifurc. Chaos 18, 1127-1149 (2008).

76. J. N. Stember and G. S. Ezra, "Fragmentation kinetics of a Morse oscillator chain under tension", Chem. Phys. 337, 11-32 (2007).

77. G. S. Ezra and S. Wiggins, "Impenetrable barriers in phase space for deterministic thermostats", J. Phys. A (Fast Track Communication) 42, 042001 (2009).

78. G. S. Ezra, H. Waalkens and S. Wiggins, "Microcanonical rates, gap times, and phase space dividing surfaces",J. Chem. Phys. 130, 164118 (2009).

79. G. S. Ezra and S. Wiggins, "Phase space geometry and reaction dynamics near index two saddles", J. Phys. A 42, 205101 (2009). 
80. A. Sergi and G. S. Ezra,, "Bulgac-Kusnezov-NoséHoover thermostats”, Phys. Rev. E 81, 036705 (2010). [cond-mat arXiv:1002.0657]

81. P. Collins, G. S. Ezra and S. Wiggins, "Phase space structure and dynamics for the Hamiltonian isokinetic thermostat", J. Chem. Phys. 133, 014105 (2010) [cond-mat arXiv:1004.4294].

82. J. N. Stember and G. S. Ezra, "Isomerization kinetics of a strained Morse oscillator ring", Chem. Phys. 381, 80-87 (2011).

83. A. Sergi and G. S. Ezra, "Algorithms for non-Hamiltonian dynamics", Atti Accad. Pelorit. Pericol. Cl. Sci. Fis. Mat. Nat., Vol. LXXXVIII, No. 2, C1C1002002 (2010).

84. G. S. Ezra and S. Wiggins, "Phase space barriers and dividing surfaces in the absence of critical points of the potential energy", preprint [chem-ph arXiv:1011.0913].

85. P. Collins, G. S. Ezra and S. Wiggins, "Index $k$ saddles and dividing surfaces in phase space, with applications to isomerization dynamics", J. Chem. Phys. 134, 244105 (2011).

86. P. Collins, G. S. Ezra and S. Wiggins, "Isomerization dynamics of a buckled nanobeam”, Phys. Rev. E 86, 056218 (2012).

87. F. A. L. Mauguiere, P. Collins, G. S. Ezra and S. Wiggins, "Bond breaking in a Morse chain under tension: fragmentation patterns, higher index saddles and bond healing", J. Chem. Phys. 138, 134118 (2013).

88. F. A. L. Mauguiere, P. Collins, G. S. Ezra and S. Wiggins, "Bifurcations of Normally Hyperbolic Invariant Manifolds in Analytically Tractable Models and
Consequences for Reaction Dynamics", Int. J. Bifurc. Chaos 23, 1330043 (2013).

89. F. A. L. Mauguiere, P. Collins, G. S. Ezra, S. C. Farantos and S. Wiggins,"Multiple Transition States and Roaming in Ion-Molecule Reactions: a Phase Space Perspective", Chem. Phys. Lett, 592, 282-287 (2014).

90. P. Collins, B. K. Carpenter, G. S. Ezra and S. Wiggins, "Nonstatistical dynamics on potentials exhibiting reaction path bifurcations and valley-ridge inflection points", J. Chem. Phys. 139:154108 (2013).

91. F. A. L. Mauguiere, P. Collins, G. S. Ezra, S. C. Farantos and S. Wiggins, "Roaming dynamics in ionmolecule reactions: phase space reaction pathways and geometrical interpretation", J. Chem. Phys. 140, 134112 (2014).

92. F. A. L. Mauguiere, P. Collins, G. S. Ezra, S. C. Farantos and S. Wiggins, Roaming dynamics in Ketene isomerization, Theoretical Chemistry Accounts 133, 1507 (2014).

93. P. Collins, Z. C. Kramer, B. K. Carpenter, G. S. Ezra and S. Wiggins, Nonstatistical dynamics on the caldera, J. Chem. Phys. 141, 034111 (2014).

94. G. S. Ezra and S. Wiggins, "Vladimir Igorevich Arnold: obituary”, Physics Today, 63 (12), 74-76 (2010).

Acknowledgments We appreciate and thank all of the authors, and the various reviewers, who contributed to this Festschrift. Finally, the enthusiastic support of Christopher Cramer and help from TCA staff is gratefully acknowledged. 